\title{
Feasibility and acceptability of conducting HIV vaccine trials in adolescents in South Africa: Going beyond willingness to participate towards implementation
}

\author{
M Wallace, ${ }^{1}$ BSocSci Hons, MSc, PhD; K Middelkoop, ${ }^{2} \mathrm{MD}, \mathrm{PhD} ;$ P Smith, ${ }^{2}$ MSocSci; C Pike, ${ }^{2}$ BSc Hons, MSc; T Bennie, ${ }^{3}$ MA; \\ J Chandia, ${ }^{4} \mathrm{MB}$ ChB, DTM\&H, DPH, DIPHC, MPraxMed, FCFP, PhD; G Churchyard, ${ }^{5} \mathrm{MB}$ BCh, FCPaed; \\ G Gray, ${ }^{6,7} \mathrm{MB}$ BCh, FCPaed, DSc; M H Latka, ${ }^{5} \mathrm{PhD}, \mathrm{MPH} ; \mathbf{M}$ Mathebula, ${ }^{8} \mathrm{MB} \mathrm{ChB}$; M Nchabeleng, ${ }^{8} \mathrm{MB} \mathrm{ChB}, \mathrm{MMed}(\mathrm{Micro})$; \\ S Roux, ${ }^{9} \mathrm{MB}$ ChB, MPH; C Slack, ${ }^{10}$ MA (Clin Psych), PhD; A Strode, ${ }^{11}$ BA, LLB, LLM; L-G Bekker, ${ }^{2}$ MB ChB, DCH, DTM\&H, FCP (SA), PhD \\ ${ }^{1}$ Cancer Association of South Africa, Cape Town, South Africa \\ ${ }^{2}$ Desmond Tutu HIV Centre, Faculty of Health Sciences, University of Cape Town, South Africa \\ ${ }^{3}$ International Partnership for Microbicides, Paarl, South Africa \\ ${ }^{4}$ HIV Vaccine Research Unit, Faculty of Health Sciences, Walter Sisulu University, Mthatha, South Africa \\ ${ }^{5}$ The Aurum Institute, Johannesburg, South Africa \\ ${ }^{6}$ Perinatal HIV Research Unit, Chris Hani Baragwanath Academic Hospital and Faculty of Health Sciences, University of the Witwatersrand, \\ Johannesburg, South Africa \\ 7 South African Medical Research Council, Johannesburg, South Africa \\ ${ }^{8}$ MeCRU Clinical Research Unit, Department of Microbiological Pathology, School of Healthcare Sciences, Sefako Makgatho Health Sciences \\ University, Pretoria, South Africa \\ ${ }^{9}$ Synexus Clinical Research SA (Pty) Ltd, Somerset West, South Africa \\ ${ }^{10}$ Vaccines Ethics Group, Discipline of Psychology, College of Humanities, University of KwaZulu-Natal, Pietermaritzburg, South Africa \\ ${ }^{11}$ Centre for Socio-Legal Studies, School of Law, University of KwaZulu-Natal, Pietermaritzburg, South Africa
}

Corresponding author: C Pike (carey.pike@hiv-research.org.za)

Background. HIV/AIDS remains a leading cause of death in adolescents (aged 15 - 25 years), and in sub-Saharan Africa HIV-related deaths continue to rise in this age group despite a decline in both adult and paediatric populations. This is attributable in part to high adolescent infection rates and supports the urgent need for more efficacious prevention strategies. In particular, an even partially effective HIV vaccine, given prior to sexual debut, is predicted to significantly curb adolescent infection rates. While adolescents have indicated willingness to participate in HIV vaccine trials, there are concerns around safety, uptake, adherence, and ethical and logistic issues.

Objectives. To initiate a national, multisite project with the aim of identifying obstacles to conducting adolescent HIV vaccine trials in South Africa (SA).

Method. A simulated HIV vaccine trial was conducted in adolescents aged 12 - 17 years across five SA research sites, using the already licensed Merck human papillomavirus vaccine Gardasil as a proxy for an HIV vaccine. Adolescents were recruited at community venues and, following a vaccine discussion group, invited to participate in the trial. Consent for trial enrolment was obtained from a parent or legal guardian, and participants aged 16 - 17 years were eligible only if sexually active. Typical vaccine trial procedures were applied during the five study visits, including the administration of vaccination injections at study visits 2,3 and 4 .

Results. The median age of participants was 14 years (interquartile range 13 - 15), with $81 \%$ between the ages of 12 and 15 years at enrolment. Overall, $98 \%$ of screened participants opted to receive the vaccine, 588 participants enrolled, and 524 (89\%) attended the final visit.

Conclusions. This trial showed that adolescents can be recruited, enrolled and retained in clinical prevention trials with parental support. While promising, these results were tempered by the coupling of sexual-risk eligibility criteria and the requirement for parental/guardian consent, which was probably a barrier to the enrolment of high-risk older adolescents. Further debate around appropriate consent approaches for such adolescents in HIV prevention studies is required.

S Afr Med J 2018;108(4):291-298. DOI:10.7196/SAMJ.2018.v108i4.12909

Adolescents (aged 15 - 24 years) remain at the centre of the HIV/ AIDS epidemic in terms of infection rates, vulnerability and impact, with the Joint United Nations Programme on HIV/AIDS (UNAIDS) reporting 4 million adolescents living with HIV globally in 2016. ${ }^{[1]}$ In eastern and southern Africa, young women and adolescent girls are disproportionately affected. Despite their recognition as a highpriority group for HIV prevention, there was only a $6 \%$ drop in new infections among adolescent females between 2010 and 2015. ${ }^{[1]}$ South Africa (SA) has one of the highest HIV prevalence rates in the world, with an estimated $18.9 \%$ of the population aged $15-49$ years infected. ${ }^{[2]}$ HIV incidence rates for women peak in the 15 - 24-year age group, while men reach peak rates 5 - 7 years later. ${ }^{[3,4]}$ Individuals who acquire HIV infection at a young age have a greater probability of transmitting the virus than those who are infected when older, making the deployment of efficacious prevention strategies to adolescent females an urgent and compelling priority. ${ }^{[5,6]}$

Vaccination is the preferred prevention method for viral infections, and HIV vaccination prior to sexual debut has been identified as a 
potential means of curbing the epidemic. ${ }^{[7]}$ Simultaneous licensure of an efficacious prophylactic HIV vaccine for both adults and adolescents is important; however, testing of a candidate vaccine in adolescent populations is likely to be required for age-specific licensure ${ }^{[8,9]}$ Compared with adults, adolescents may exhibit different vaccination-induced immune responses and safety profiles, and show differences in vaccine uptake and effectiveness. ${ }^{[8]}$ While vaccine safety and efficacy is a biomedical concern, broader issues relating to adolescent participation in clinical trials must be considered, including uptake, ethical, sociobehavioural and logistic challenges. ${ }^{[9-15]}$

To date, no clinical HIV vaccine trials have involved adolescents, although a number of preparatory and acceptability studies have been reported. This work has focused on the acceptability of a preventive HIV vaccine ${ }^{[16-18]}$ and of adolescent participation in vaccine trials. ${ }^{[16,19-24]}$ Proposed models of adolescent inclusion in HIV vaccine trials suggest that the eligibility age for study participants may be rolled down to 16 - 18 years once safety and immunogenicity are established in adult efficacy trials, along with phase $1-2$ bridging safety and immunogenicity trials for younger age groups. Research conducted in SA indicates that hypothetical willingness for adolescent participation in vaccine trials is high for various reasons, but concerns remain. ${ }^{[1,20,22,23,25]}$ Several studies have also focused on issues relating to feasibility of research in adolescents, including the viability of recruiting high-risk adolescents for efficacy trials. Most have used observational cohorts where the intervention is HIV testing and incidence and retention are the main outcomes. ${ }^{[26-29]}$ While these studies provide useful preliminary data, it is important to note their limitations. Attitudes alone are unlikely to predict behaviour, and the value of examining 'willingness to participate' in a vaccine trial and its predictors is therefore limited. ${ }^{[29]}$ Additionally, recruiting an observational cohort may not adequately reflect challenges arising from providing an experimental product to adolescents in a clinical setting.

\section{Objectives}

In order to develop an authentic understanding of the issues related to running HIV vaccine trials in adolescents, we initiated a national, multisite project with the broad objective of identifying clinical, community, ethical, legal and sociobehavioural obstacles to the conduct of adolescent HIV vaccine trials in SA and building capacity to address these, through the enactment of a simulated HIV vaccine trial. This study used the Merck human papillomavirus (HPV) vaccine Gardasil, already licensed but not yet available at the time, as a proxy for an HIV vaccine in adolescents in SA. The use of a registered vaccine with established safety and immunogenicity enabled focus on key feasibility questions such as recruitment, consent, retention, uptake and completion of a vaccine schedule; risk behaviours and HIV, sexually transmitted infection (STI) and pregnancy incidence in the cohort; and exploration of models for adolescent inclusion in HIV vaccine trials.

\section{Methods}

The study was a multisite, open-label longitudinal cohort of 12 17-year-old, HIV-negative male and female adolescents. Sites at five SA HIV research organisations participated in this study: Desmond Tutu HIV Foundation, Cape Town; CAPRISA (Centre for the AIDS Programme of Research in South Africa), Durban; Perinatal HIV Research Unit, Soweto; MeCRU Clinical Research Unit, Pretoria; and The Aurum Institute, Rustenberg. These research sites are located in low-income, rural and urban areas with high HIV incidence rates. Adolescents were recruited through community outreach by targeting schools, youth groups, taxi ranks and other points of community convergence. Adolescents were also reached through their parents via outreach recruitment leaflets and activities.

Throughout, procedures simulating those of a typical HIV vaccine trial were adhered to as far as possible. Adolescents and parents were invited to attend vaccine discussion groups (VDGs) where information was provided on study procedures (including randomisation and blinding concepts to replicate an actual vaccine trial), HPV and the HPV vaccine. Following the VDG, attendees were invited to attend a screening visit. The age of consent to health research in SA is 18 years, and as the age eligibility criterion was 17 at screening, all potential participants were therefore required to sign assent forms after their parent/guardian signed a consent form for their child's enrolment. Both completed a questionnaire that assessed understanding of the study objectives and procedures, where continuation of study participation was dependent on achievement above a minimum score threshold. This assessment of understanding was re-administered to participants at the end of the study (month 9).

All participants were required to be HIV-negative at screening, and females could not be pregnant or breastfeeding. In order to simulate the potential requirements of an HIV vaccine trial, participants aged $\geq 16$ years (the age at which adolescents can lawfully consent to sex in SA) were required to be sexually active (defined as ever having had vaginal or anal sex). Sexual debut was not required as an eligibility criterion in youth aged 12 - 15 years. Sexually active females were encouraged to use hormonal and/or barrier contraception throughout the vaccine schedule, and both contraceptive methods were provided to participants. To safeguard adolescent privacy, parents/guardians were clearly told which clinical information they would and would not be party to as the trial continued. ${ }^{[30,31]}$ Adolescents meeting the study criteria were enrolled and opted (with parental/guardian consent) to receive the HPV vaccine or not. Both those receiving and those not receiving the vaccine underwent identical study procedures apart from vaccine administration, such as perceived risks and benefits, age, gender, and knowledge of disease and vaccine, in order to determine correlates between vaccine uptake and refusal.

Participants attended five visits in total: screening, enrolment within 45 days of screening (first administration of vaccine), 3 months (second administration of vaccine), 6 months (final administration of vaccine), and 9 months (final follow-up including an assessment of study understanding). At each visit, HIV counselling and testing, syphilis screening and pregnancy testing for females were conducted and sexual risk behaviour was assessed. STI symptoms were recorded at every visit. HIV testing utilised rapid finger-prick antibody-based tests and followed national HIV testing guidelines, ${ }^{[32]}$ while an RPR (rapid plasma reagin) test was used to screen for syphilis. The HPV vaccine schedule was paused for females who became pregnant after enrolment, but was continued for participants who seroconverted during the study. Sexual risk behaviour was assessed through a quantitative questionnaire designed specifically for this study and administered by research staff in the participant's home language.

\section{Ethical considerations}

Ethical approval was obtained from the human research ethics committees of the University of Cape Town (ref. no. 245/2008), the University of the Witwatersrand (ref. no. 080710), the University of KwaZulu-Natal (ref. no. BF109/08) and the University of Limpopo (ref. no. MREC/P/110/2009). When study procedures identified HIV infection, steps were taken to counsel participants, ensure timely participant disclosure to a trusted adult, and link participants to care. 
Efforts were made to reduce the risk of HIV infection of enrolled participants, and assistance was provided to participants with syphilis or those who became pregnant while enrolled. There was engagement with participating community representatives, including community advisory board youth representatives, as recommended for adolescent prevention trials. ${ }^{[31-37]}$

\section{Statistical analysis}

Data were analysed using Stata 14.0 (StataCorp, USA). Participants were divided into two age groups, using age at enrolment (12 15 and $16-17$ years), based on the different inclusion criteria regarding sexual activity. The assessment of understanding score was calculated as the number of correct answers to 13 questions. Bivariate analysis employed Student's $t$-test, Fisher's exact test and the $\chi^{2}$ test of proportions, as appropriate; for intra-individual comparisons of measures taken at baseline and 9 months, McNemar's $\chi^{2}$ test, McNemar's exact test and paired $t$-tests were used. The incidences of pregnancy and HIV seroconversion were calculated as the number of incidence events divided by total person-time of follow-up. Survival analyses, including Kaplan-Meier tables and Cox's proportional hazards models, were used to determine factors associated with loss to follow-up in the cohort. All statistical tests were two-sided at $\alpha=0.05$. Owing to delayed starts and study funding contracts, the study procedures were halted for a number of participants (mainly from two sites) prior to their scheduled follow-up completion. These individuals completed their vaccine schedules, but no further study data were collected. Analyses of retention (defined as completion of all five study visits), vaccine completion and follow-up behaviours excluded these participants.

\section{Results}

\section{Cohort description}

Screening and enrolment took place from November 2009 to October 2010, with staggered site initiation and mean recruitment of 168 participants per site over 8.4 months (Fig. 1). Of the 961 adolescents screened across the five sites, 844 (87.8\%) were enrolled (Table 1). The majority of adolescents who enrolled were in the 12 - 15-yearold age group (81\%). The median age of enrolled participants was 14 years (interquartile range (IQR) 13 - 15) at screening, and $61 \%$ were female. The mean assessment of understanding score at enrolment was 11.3 out of 13 (IQR 11 - 12). The most common reasons for failure of screening across all sites were adolescents

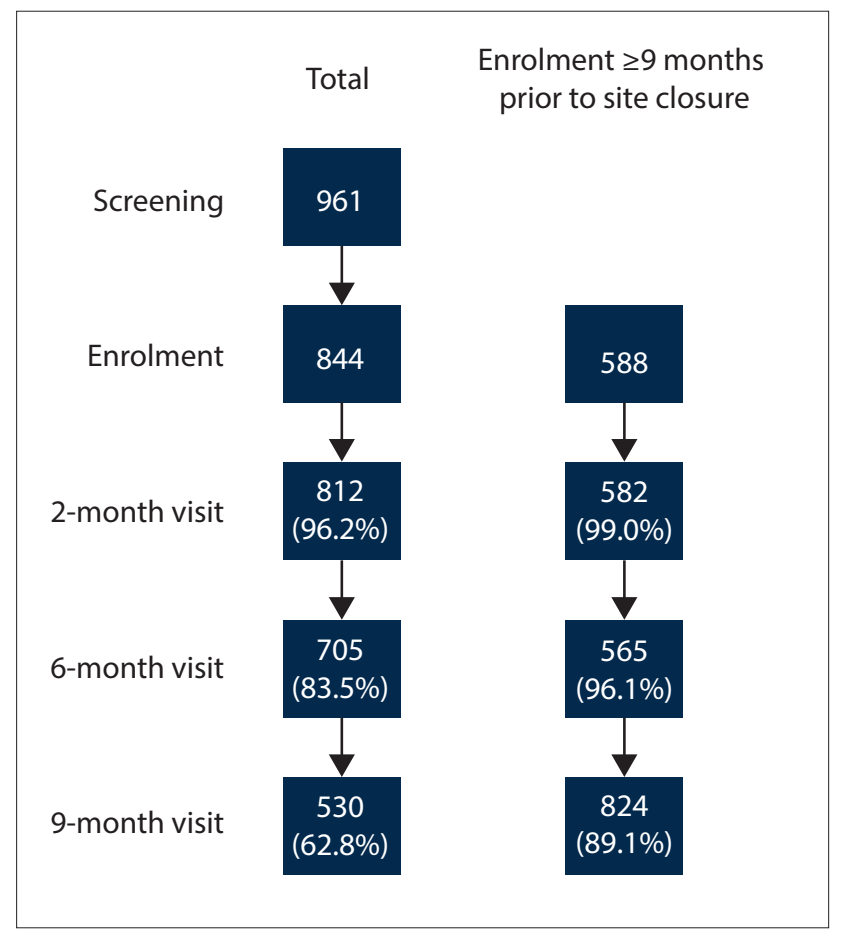

Fig. 1. Participant visit attendance and retention by site.

\begin{tabular}{|c|c|c|c|c|}
\hline \multirow[b]{2}{*}{ Variable } & \multicolumn{2}{|c|}{ Age (years), $n(\%)$} & \multirow[b]{2}{*}{ Total $(N=843)$} & \multirow[b]{2}{*}{$p$-value } \\
\hline & $\begin{array}{l}12-15(N=684, \\
81 \%)\end{array}$ & $\begin{array}{l}16-17(N=159, \\
19 \%)\end{array}$ & & \\
\hline Gender female, $n(\%)$ & $420(61)$ & $90(57)$ & $510(61)$ & 0.27 \\
\hline Ever had sex, $n(\%)$ & $58 / 674(9)$ & $135 / 157(86)$ & $193 / 831(23)$ & $<0.001$ \\
\hline Age (years) at first sex, median (IQR) & $\begin{array}{l}14(12-15) \\
(n=56)\end{array}$ & $\begin{array}{l}15(15-16) \\
(n=135)\end{array}$ & $\begin{array}{l}15(14-16) \\
(N=191)\end{array}$ & $<0.001$ \\
\hline Number of sexual partners, median (IQR) & $\begin{array}{l}1(1-1) \\
(n=53)\end{array}$ & $\begin{array}{l}1(1-2) \\
(n=132)\end{array}$ & $\begin{array}{l}1(1-2) \\
(N=185)\end{array}$ & 0.16 \\
\hline Of all the times had sex, frequency of condom use, $n(\%)$ & & & & 0.002 \\
\hline Always & $30 / 57(53)$ & $94 / 135(70)$ & $124 / 192(64)$ & \\
\hline Usually & $5 / 57(9)$ & $5 / 135(4)$ & $10 / 192(5)$ & \\
\hline About half the time & $3 / 57(5)$ & $16 / 135(12)$ & $19 / 192(10)$ & \\
\hline Rarely & $1 / 57(2)$ & $6 / 135(4)$ & $7 / 192(4)$ & \\
\hline Never & $18 / 57(32)$ & $14 / 135(10)$ & $32 / 192(17)$ & \\
\hline \multicolumn{5}{|l|}{ Ever engaged in transactional sex, $n(\%)$} \\
\hline Received & $2 / 55(4)$ & $2 / 135(1.5)$ & $4 / 190(2)$ & 0.58 \\
\hline Given & $1 / 57(2)$ & $3 / 133(2)$ & $4 / 190(2)$ & 1.00 \\
\hline Ever had sex with someone without knowing their HIV status, $n$ (\%) & $32 / 56(57)$ & 79/134 (59) & $111 / 190(58)$ & 0.82 \\
\hline Has someone ever used force to make you have sex, $n(\%)$ & $6 / 57(11)$ & $11 / 134(8)$ & $17 / 191(9)$ & 0.61 \\
\hline Ever had an STI, $n(\%)$ & $2 / 56(4)$ & $3 / 129(2)$ & $5 / 185(3)$ & 0.64 \\
\hline Ever had sex while drunk or under the influence of a drug, $n(\%)$ & $3 / 57(5)$ & $16 / 132(12)$ & $19 / 189(10)$ & 0.19 \\
\hline
\end{tabular}


aged 16 - 17 years not being sexually active ( $n=46$ participants) or participants being lost to follow-up after arriving without a biological parent or legal guardian and then never returning or not being contactable afterwards $(n=273)$.

Owing to delayed starts at the sites and study funding contracts, 256 participants (30\%) were not enrolled in time to finish the full five visits. When those who completed the full 9 months of study time were compared with those who enrolled late, these participants did not differ in terms of age, gender or reporting at baseline ever having had sex. Baseline data from all 843 participants are presented in Table 1. Participants who could not complete 9 months of follow-up owing to termination of study procedures $(n=256)$ were excluded from assessments of retention and vaccine completion (Fig. 2). This early closure mainly affected two sites.

\section{Sexual risk behaviour and assessment of understanding}

Table 1 shows baseline sexual risk behaviour of participants as reported in self-administered questionnaires by the two eligibility age groups, $12-15$ and $16-17$ years. Of the participants, 23\% reported ever having had sex, of whom $9 \%$ were 12 - 15 years old. Despite the

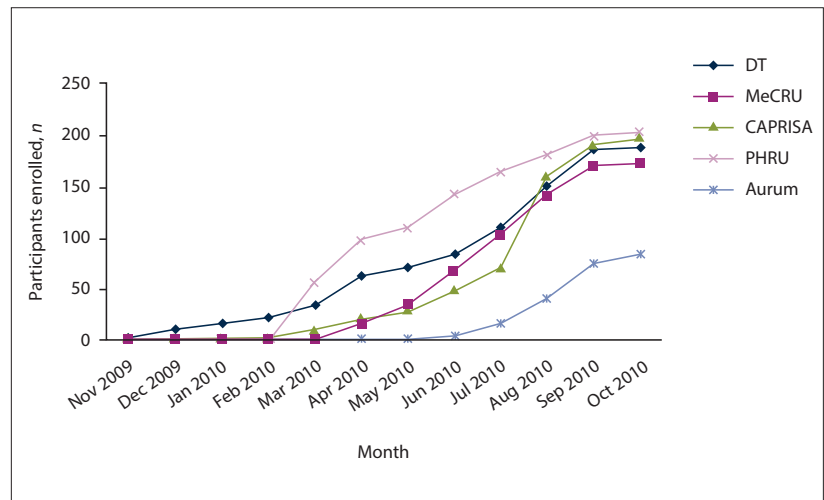

Fig. 2. Cumulative number enrolled by site: Desmond Tutu HIV Foundation (DT), Cape Town; MeCRU Clinical Research Site, Pretoria; Centre for the AIDS Programme of Research in South Africa (CAPRISA), Durban; Perinatal HIV Research Unit (PHRU), Soweto, Johannesburg; and The Aurum Institute, Rustenburg. eligibility requirement, only $86 \%$ of 16 - 17-year-olds reported sexual activity at baseline. The median age of sexual debut was lower in the younger age group ( 14 v. 15 years; $p<0.0001)$. Consistent condom use increased with age, with 12 - 15-year-old sexually active participants significantly less likely to have used condoms than 16 - 17-yearolds ( $10 \%$ v. $32 \%$, respectively; $p=0.002)$. There were no statistically significant differences across other baseline sexual risk indicators between the age groups.

Table 2 reports changes in sexual risk behaviour from baseline to study end. This analysis was restricted to those participants who attended the study visit at month 9 , and mainly focused on questions regarding sexual activity 'in the past 6 months'. Within the age groups, the only statistically significant change was a reported increase in the number of 12 - 15-year-olds ever having had sex $(p=0.01)$. However, as the numbers of match pairs reporting sexual activity were small, we also assessed changes across all participants at baseline and those retained at 9 months. Borderline improvement was noted in the proportions of participants who reported using condoms 'always' or 'usually' from baseline to study end ( $74 \%$ to $86 \%$; $p=0.05$ ), while the number who reported 'never' using a condom fell significantly $(12 \%$ to $1.4 \% ; p=0.001$ ). Compared with baseline, at the end of the study more participants reported having had sex with someone known to be HIV-positive ( $0 \%$ to $4 \% ; p=0.03$ ). Mean assessment of study procedure understanding scores decreased from 11.3 at baseline to 11.1 at 9 months (paired $t$-test: $p=0.001$ ), and this decrease was noted across both age groups.

\section{Cohort retention}

Fig. 3 depicts a Kaplan-Meier curve of participant retention over the study period, stratified by the two age groups. Considering only the participants who had the potential to complete all five sessions before study termination, overall retention (defined as attendance at all five study visits) was $89 \%$. There was no difference in retention between the two groups ( $88 \%$ in the younger group v. $87 \%$; log-rank test: $p=0.55)$. Retention was not associated with gender or with reporting being sexually active at baseline (Table 3); however, attrition had a borderline association with baseline reports of having ever had sex while drunk or under the influence of drugs $(p=0.06)$ (Table 3 ). There was no association between baseline assessment of understanding

Table 2. Change in sexual risk behaviour over time (enrolment to study end in participants retained for 9 months)

\begin{tabular}{|c|c|c|c|c|c|c|c|c|}
\hline \multirow[b]{3}{*}{ Variable } & \multicolumn{8}{|c|}{ Age (years) } \\
\hline & \multicolumn{4}{|c|}{$12-15(N=422)$} & \multicolumn{4}{|c|}{$16-17(N=100)$} \\
\hline & $N$ & Baseline & 9 months & $p$-value & $N$ & Baseline & 9 months & $p$-value \\
\hline Ever had sex, $n(\%)$ & 422 & $33(8)$ & $48(11)$ & 0.001 & 100 & $87(87)$ & $87(87)$ & 1.00 \\
\hline Had sex in past 6 months, $n(\%)$ & 24 & $9(38)$ & $10(42)$ & 1.00 & 74 & $47(64)$ & $43(58)$ & 0.54 \\
\hline Frequency of condom use in the past 6 months, $n(\%)$ & 8 & & & & 37 & & & 0.50 \\
\hline Always & & $4(50)$ & $5(63)$ & & & $28(76)$ & $27(73)$ & \\
\hline Usually & & $2(25)$ & $2(25)$ & & & $0(0)$ & $4(11)$ & \\
\hline About half the time & & $2(25)$ & $0(0)$ & & & $6(16)$ & $5(14)$ & \\
\hline Rarely & & $0(0)$ & $1(13)$ & & & $1(3)$ & $1(3)$ & \\
\hline Never & & $0(0)$ & $0(0)$ & 1.00 & & $2(5)$ & $0(0)$ & \\
\hline \multicolumn{9}{|l|}{ In the past 6 months have you had, $n(\%)$} \\
\hline \multicolumn{9}{|l|}{ Transactional sex } \\
\hline Received & 7 & $0(0)$ & $0(0)$ & 1.00 & 35 & $2(61)$ & $3(9)$ & 1.00 \\
\hline Given & 8 & $0(0)$ & $0(0)$ & 1.00 & 34 & $1(3)$ & $2(6)$ & 1.00 \\
\hline Sex with someone known to be HIV positive & 8 & $0(0)$ & $0(0)$ & 1.00 & 35 & $0(0)$ & $1(3)$ & 1.00 \\
\hline Sex with someone without knowing their HIV status & 8 & $4(50)$ & $4(50)$ & 1.00 & 36 & $19(53)$ & $13(36)$ & 0.29 \\
\hline Sex while drunk or under the influence of a drug & 7 & $1(14)$ & $0(0)$ & 1.00 & 35 & $3(9)$ & $5(14)$ & 0.73 \\
\hline
\end{tabular}




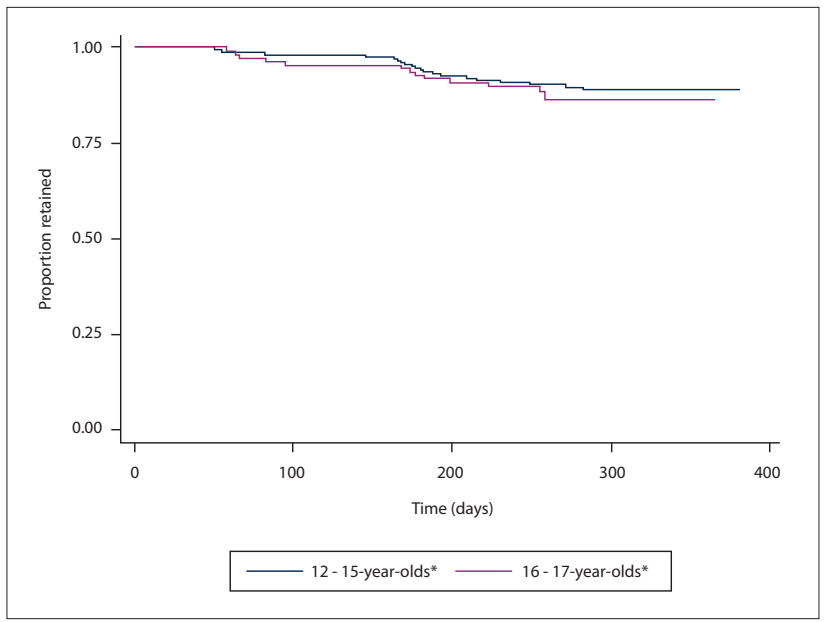

Fig. 3. Kaplan-Meier graph of retention, stratified by age group. $\left({ }^{*}\right.$ Age at screening. $)$

scores and attrition $(p=0.94)$. Reasons why some participants were lost to follow-up were not recorded.

\section{HIV, STI and pregnancy incidence}

Ten pregnancies occurred over the study period, with a total of 511.6 years of follow-up time accumulated across all five sites, giving an incidence rate of 1.95 per 100 person-years (95\% confidence interval (CI) 0.9 - 3.6). Five of the 10 pregnant participants reported contraceptive use at enrolment, 3 using male condoms, 3 using the female condom, and 3 using injectable contraceptives (one participant used two of these methods, and one three). Females who had their sexual debut prior to study participation were more likely to become pregnant (odds ratio 5.09, 95\% CI $1.4-18.21$ ). Six of the 10 participants who became pregnant prior to the 6- or 9-month appointment were lost to follow-up.

The trial was conducted in areas with a high HIV burden, and HIV seroconversion occurred in two participants, although one participant seroconverted between screening and enrolment, suggesting that she was in the window period at screening. Based on the one incident infection, which was detected at month 9, the incidence was 0.20 per 100 person-years (95\% CI 0.005 - 1.1). Both seroconverters were female, aged 13 and 15 years. Syphilis testing was conducted at each study visit and overall only one participant tested positive for syphilis, at the time of enrolment.

\section{Vaccinations}

Overall, 826 (98\%) of participants with parental/guardian consent agreed to receive the HPV vaccine. Of the 580 participants who were consented and enrolled at least 9 months prior to study termination, 532 (92\%) completed the full HPV vaccination schedule.

\section{Discussion}

This study proved the feasibility of recruiting and retaining adolescents in a clinical trial in which a multidose 'STI' vaccine was administered to participants, emulating an adolescent HIV vaccine trial. This study supports a model of adolescent inclusion in HIV vaccine trials where, once a potential vaccine regimen is considered safe and immunogenic in adult efficacy studies, the eligibility age for study participation may be 'rolled down' to $16-18$ years in order to be inclusive of younger participants (shown in Fig. 4). ${ }^{[21,33]}$ Phase 1 - 2 bridging studies for safety and immunogenicity can then be designed for younger adolescent age groups in whom sexual activity has not yet commenced. Minor adolescent participants could then contribute to safety and immunogenicity data without contributing to efficacy data.

To date, HIV prevention trials have targeted adults at high risk for HIV acquisition by enrolling individuals who have multiple sex partners, partners of unknown or positive HIV serostatus, or sex without condoms. In this study, we asked only that older adolescents (aged $16-17$ years) be sexually active, as 16 is the age of consent to sex in SA and inclusion of such a criterion would have had legal implications. Sexual activity is no longer a reportable offence when younger adolescents engage in sex with peers, or with an older partner where the age difference is less than 2 years; however, parental and societal pressure remains a barrier to high-risk adolescents acknowledging sexual activity and taking part in such trials when parental consent is required. ${ }^{[35,38]}$ Although 961 adolescents were recruited in this study, sexual eligibility criteria combined with the need for parental consent may have influenced the age distribution, as the majority had not initiated sexual activity and only $19 \%$ of the enrolled sample fell within the 16 - 17-year age group. There is a need for study investigators, institutional ethics bodies and regulators to consider alternative approaches for the inclusion of older adolescents that do not involve parental consent.

The trial was modelled on previous adolescent HPV vaccine licensure studies, and in order to mimic the likely consent approach for an HIV vaccine trial, participants in our study required parental/ guardian consent to enrol. In the VDGs, the HPV vaccine was portrayed as a vaccine to prevent a sexually transmitted infection in order to fit with a simulated HIV vaccine design. At the time of protocol design, consultations and discussions were held around the dynamic ethicolegal framework of SA research, laws and involvement of minors, and these discussions have led to a number of important publications. ${ }^{[31,34-37]}$ This study suggests that the requirement for

\begin{tabular}{lcc} 
Table 3. Bivariate association of baseline demographics and sexual risk behaviours with attrition & Bivariate analysis \\
\hline & HR (95\% CI) & $p$-value \\
Variable & $1.09(0.93-1.29)$ & 0.25 \\
\hline Age at enrolment & $0.59(0.25-1.39)$ & 0.66 \\
Gender female & $1.36(0.67-2.384)$ & 0.29 \\
Ever had sex & & 1.00 \\
Ever engaged in transactional sex & - & 1.00 \\
$\quad$ Received & - & 0.86 \\
$\quad$ Given & $0.92(0.34-2.41)$ & 0.14 \\
Ever had sex with someone without knowing their HIV status & $3.05(0.70-13.33)$ & 0.06 \\
Has someone ever used force to make you have sex & $2.97(0.97-9.12)$ & \\
Ever had sex while drunk or under the influence of a drug & & \\
HR = hazard ratio; CI = confidence interval. & &
\end{tabular}




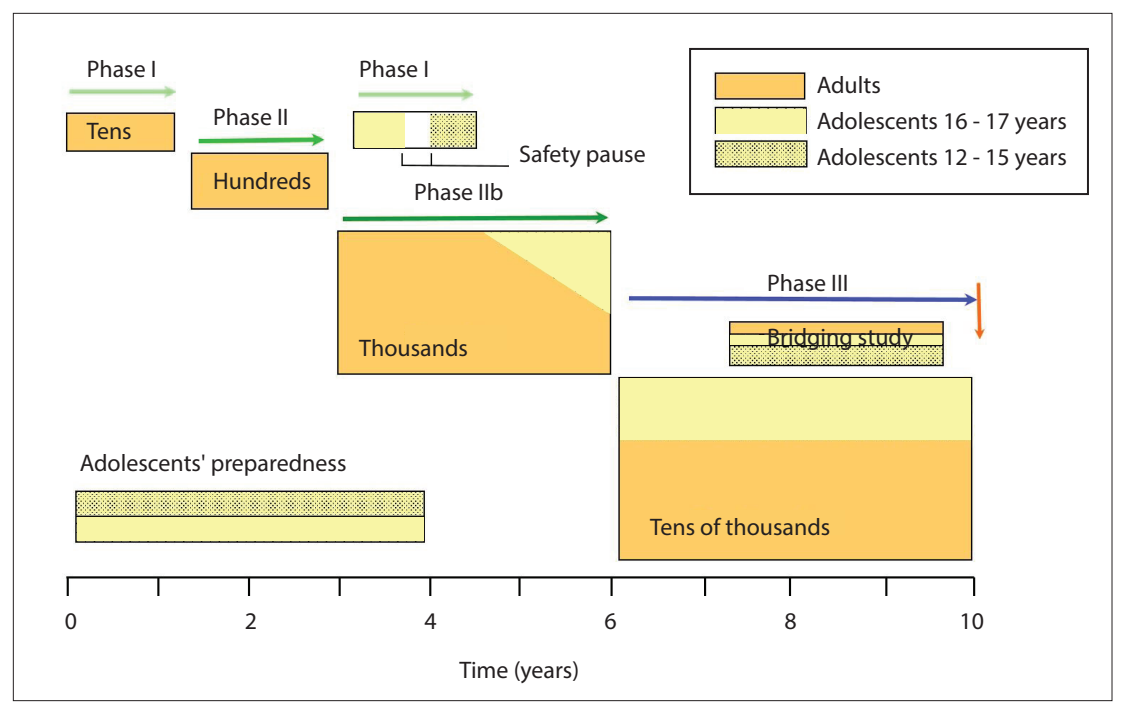

Fig. 4. A modelled approach to introducing adolescent clinical vaccine trials over time, adapted from that proposed by Lau et al. ${ }^{[33]}$

parental/guardian consent coupled with the requirement for older adolescents to be sexually active may have been an obstacle to recruitment of high-risk adolescents, owing to awareness of sexual activity on the part of the parents/guardians and resultant discomfort between the adults and the adolescents. Had a proportion of participants in the 16 - 17-year-old age group been prestipulated, recruitment may have been more challenging. Going forward, this 'dilution of incidence' effect should be catered for by limiting the number of adolescents in the adult trial (Fig. 4) and/or factoring in a lower incidence contribution from this age group. Alternatively, ways should be explored to identify high-risk young individuals without requiring disclosure of behaviours to guardians.

\section{HIV incidence}

Overall, self-reported sexual risk behaviour at study onset was low, and HIV incidence was particularly low relative to SA standards at 0.20 per 100 person-years. This is lower than the estimated incidence of $0.56 / 100$ personyears in 15 - 18-year-olds mathematically derived from prevalence data by Shishana $e t$ al. ${ }^{[39]}$ as well as lower than that observed in a 16 - 20-year-old cohort study in a similar setting $\left(9.2 / 100\right.$ person-years). ${ }^{[22]}$ It also contrasts with vaccine preparedness studies, where behavioural data suggest that individuals volunteering for vaccine-related research may be more likely to be at risk of HIV than those who do not volunteer. Smit et al. ${ }^{[24]}$ compared risk profiles of individuals volunteering for an HIV vaccine preparedness cohort with those of a community sample. Adjusting for age, gender, education and risk perception, cohort participants were younger, more likely to have been treated for an STI, and less likely to use condoms. Jaspan et al. ${ }^{[20]}$ also found high risk levels in an 11 - 19-year-old adolescent cohort, with one-third having experienced sexual debut (at a mean age of 14.6 years) and an HIV prevalence rate of $10.6 \%{ }^{[20]}$ In a 12 -month follow-up of 14 - 17-year-olds, Jaspan et al. ${ }^{[40]}$ found reports of high-risk behaviours and, the occurrence of five pregnancies supported reports of unprotected sex..$^{[40]}$ Parental/ guardian consent was waived in this latter study. Furthermore, participation of adolescents in this study who were sexually active with full parental knowledge may imply that they were more self-efficacious individuals, and so more likely to engage in safer sex practices.

\section{Sexual risk behaviour}

The study findings highlight the challenges of collecting accurate, authentic sexual risk behaviour data from adolescents. The discrepancy between the eligibility requirement for sexual activity in 16 - 17-year-olds and the reporting of this behaviour in only $87 \%$ of the cohort may reflect either misunderstanding by some adolescents or inaccuracies around behaviour reporting at the time of enrolment or questionnaire completion. Recent prevention trials in adult women in SA have highlighted that a strong reason for individual participation is the access to improved health services that clinical research offers. In this case, access to an otherwise unavailable vaccine may have prompted young people to report behaviours inaccurately.

At baseline, reported condom use improved with increasing age. This finding suggests that adolescents become more although no HIV seroconversions occurred, skilled at negotiating condom use as they become more sexually experienced, while younger adolescents may be less prepared for or anticipating of sex. However, sexual activity in the youngest age group was infrequent and results from this group should be interpreted with caution. By the end of the study, improvements in condom use were observed, which may be due to increasing age, preparedness for and experience with sex (as before) or to study participation, as risk reduction counselling, HIV testing and condoms were provided at every visit.

In addition, by the end of the study more participants reported knowing their partners' serostatus. This may be a function of more prevalent testing as adolescents get older and begin to engage with older partners, or also a result of study participation. Risk behaviours are studied repeatedly throughout prevention trials because the concern remains that participation may cause sexual disinhibition, where individuals exhibit increased risk behaviour owing to perceived protection. This concern is repeatedly raised in the context of youth involvement in clinical prevention trials. However, clinical trials in adults have consistently shown reduced risky behaviour during trial participation, and although adolescents are more challenging to study, owing to natural developmental and behaviour changes, our data showed no evidence of behavioural disinhibition.

In contrast to HIV rates, the pregnancy incidence in this cohort was relatively high. This suggests the practice of condomless sex coupled with age-related lower HIV risk networks. It is possible that young women are on the brink of exposure to sexual partners who will place them at risk of HIV infection as well as pregnancy, again emphasising the urgent need for a protective vaccine before this occurs.

\section{Trial retention}

Retention in the study was $89 \%$. Previous studies reported retention rates of $87 \%$ in a 16 - 20-year-old cohort $^{[22]}$ and $82 \%$ in a 14 - 17-year-old cohort, ${ }^{[40]}$ each followed for 12 months. Adolescence is a challenging age for trial retention owing to competing priorities, busy schedules and structural impediments, as well as evolving autonomy and independence. Capacity building was initially undertaken to ensure that all trial sites were competent to enrol adolescents and that all would be safe, friendly and attractive to adolescents. ${ }^{[41]}$ Perception of the value of the HPV vaccine, both costly and at the time inaccessible to most SA adolescents, may have contributed to the high retention 
rate. Albeit unavailable, the vaccine used was known to be safe and effective, and this may have contributed to the high retention rate. It is not known how willingness of this age group to participate would have been affected if an experimental vaccine product of unknown efficacy had been offered.

Although the marginal decrease in assessment of understanding scores across the study period was statistically significant, we doubt that it is clinically significant. Regardless, this is a reminder that informed consent is an ongoing process that should be informally evaluated at every study visit. While vaccine discussion groups can bolster understanding, the way in which information is initially imparted and ongoing reminders of details over time are particularly important for long clinical trials. This may be especially true for adolescents, who can have difficulty in grasping information, and concepts should be revisited to ensure ongoing informed consent.

It is worth noting here that SA now conducts a national HPV vaccination programme in adolescents, and HPV vaccine uptake and completion rates have been excellent and above those reported from other countries. ${ }^{[42,43]}$ Qualitative focus group research in SA had highlighted the concerns of mothers regarding agreement to HPV vaccination of their daughters, ${ }^{[44]}$ yet this did not appear to affect behaviour in the present study, with $98 \%$ of participants and their parents opting to receive the HPV vaccine. This is remarkable, because the HPV vaccine was framed in this study as a means to prevent STI, rather than the more palatable cancer prevention message.

This study augurs well for an extended vaccine schedule in adolescents, providing useful information both for HPV vaccine rollout in SA and for planning future adolescent HIV vaccine trials. ${ }^{[45,46]}$ This is particularly relevant in the context of the phase IIb/III HVTN 702 vaccine trial in SA, which will evaluate the efficacy and safety of a clade C ALVAC HIV and bivalent subtype C gp120/MF59 vaccine. Success in the phase IIb/III trial will pave the way for extension of the trial to adolescent participants. If the adolescent trial is successful, there could be potential for an effective, school-based, dual HPV/ $\mathrm{HIV}$ vaccine programme in SA.

\section{Conclusions}

The use of an HPV vaccine as a surrogate for an HIV vaccine has shown that adolescents aged 12 - 17 years can be successfully and easily recruited, enrolled and retained in clinical prevention trials in both rural and peri-urban SA, including trials framed as STI prevention (Fig. 3). In this study, the parental/guardianship consent strategy, combined with sexual-risk eligibility criteria, may have acted as a barrier to the enrolment of older high-risk adolescents. This suggests that HIV prevention studies enrolling high-risk, sexually active adolescents must carefully consider the most appropriate consent approach. National ethical guidelines allow a waiver of parental/guardianship consent for adolescent studies where specific criteria are met, including that study procedures pose minimal risk. SA is currently enrolling adults only into the first HIV vaccine licensure study. ${ }^{[47]}$ It is clear that should this vaccine be even partially efficacious, the question of licensure in adolescents will be key. One option for HIV vaccine trials is that minor adolescent participants could contribute safety and immunogenicity data only without contributing to efficacy data, but there may be alternative approaches to include older at-risk adolescents that will require the consent strategy to be discussed with institutional ethics bodies and the regulators who ultimately license the product.

Acknowledgements. We acknowledge the importance of a capacitybuilding grant from the Bill and Melinda Gates Foundation together with the European and Developing Countries Clinical Trials Partnership (EDCTP). The grant allowed the successful building of capacity for safe and effective adolescent clinical trials in six SA sites. We also sincerely thank Merck Sharp \& Dohme (MSD) for their generous donation of Gardasil HPV vaccine sufficient for all the adolescents in the study. We also thank all the clinical teams and terrific study co-ordinators, who made the experience safe and friendly for the participants. Finally, we acknowledge all the young South Africans and their guardians who helped to contribute to this work by volunteering.

Author contributions. Conception, design and site oversight: LGB, GG, GC, CS, AS; collection of data and assembly: MW, MHL, TB, MM, MN; data analysis and interpretation: KM, PS; manuscript writing and editing: all authors; final approval of manuscript: all authors

Funding. Funding for this work was provided by the EDCTP (grant no. CT.2006.33111.004) and the Bill \& Melinda Gates Foundation.

Conflicts of interest. None. MSD was not involved in the design, analysis or final preparation of the manuscript.

1. Joint United Nations Programme on HIV/AIDS (UNAIDS). Global AIDS Update 2016. Geneva: UNAIDS, 2016.

2. Statistics South Africa. Community Survey 2016. Statistical release P0301. Pretoria: Stats SA, 2016.

3. Hallett TB, Stover J, Mishra V, et al. Estimates of HIV incidence from household-based prevalence surveys. AIDS 2010;24(1):147-152. https://doi.org/10.1097/QAD.0b013e32833062dc

4. Rehle TM, Hallett TB, Shisana O, et al. A decline in new HIV infections in South Africa: Estimating HIV incidence from three national HIV surveys in 2002, 2005 and 2008. PLoS One 2010;5(6):el1094. https://doi.org/10.1371/journal.pone.0011094

5. Johnson LF, Dorrington RE, Bradshaw D, et al. Sexual behaviour patterns in South Africa and their Johnson LF, Dorrington RE, Bradshaw D, et al. Sexual behaviour patterns in South Africa and their
association with the spread of HIV: Insights from a mathematical model. Demogr Res 2009;21(11):289association with the spread of HIV: Insights from a

6. Bershteyn A, Klein DJ, Eckhoff PA. Age-dependent partnering and the HIV transmission chain: A microsimulation analysis. J R Soc Interface 2013;10(88):20130613. https://doi.org/10.1098/ rsif. 2013.0613

7. McClure CA, Gray G, Rybczyk G, Kyle G, Wright P. Challenges to conducting HIV preventative vaccine trials with adolescents. J Acquir Immune Defic Syndr 2004;36(2):726-733. https://journals. lww.com/jaids/Abstract/2004/06010/Challenges_to_Conducting_HIV_Preventative_Vaccine.10.aspx (accessed 13 March 2018)

8. Rudy BJ, Kapogiannis BG, Lally MA, et al. Youth-specific considerations in the development of PrEP microbicide and vaccine research trials. J Acquir Immune Defic Syndr 2010;54(Suppl 1):S31-S42. https://doi.org/10.1097/QAI.0b013e3181e3a922

9. Tsu VD, Cernuschi T, LaMontagne DS. Lessons learned from HPV vaccine delivery in low-resource settings and opportunities for HIV prevention, treatment, and care among adolescents. J Acquir Immune Defic Syndr 2014; 66(Suppl 2):S209-S216. https://doi.org/10.1097/QAI.0000000000000175 10. DiClemente RJ, Ruiz MS, Sales JM. Barriers to adolescents' participation in HIV biomedical prevention research. J Acquir Immune Defic Syndr 2010;54(Suppl 1):S12-S17. https://doi.org/10.1097/
QAI.0b013e3181ele2c0

QA1.0Bolsesistele2

1. Hosek SG. Zimet GD. Behavioural considerations for engaging youth in HIV clinical research. J Acquir Immune Defic Syndr 2010;54(Suppl 1):S25-S30. https:///oi.org/10.1097/QAI.0b013e3181e15c22

12. Moodley N, Gray G, Bertram M. The case for adolescent HIV vaccination in South Africa: A cost-effectiveness analysis. Medicine (Baltimore) 2016;95(4):e2528. https://doi.org/10.1097/ MD. 0000000000002528

13. Nelson RM, Lewis LL, Struble K, Wood SF. Ethical and regulatory considerations for the inclusion of adolescents in HIV biomedical prevention research. J Acquir Immune Defic Syndr 2010;54(Suppl 1):S18-S24. https://doi.org/10.1097/QAI.0b013e3181e2012e

4. Pace JE, Siberry GK, Hazra R, Kapogiannis BG. Pre-exposure prophylaxis for adolescents and young adults at risk for HIV infection: Is an ounce of prevention worth a pound of cure? Clin Infect Dis 2013;56(8):1149-1155. https://doi.org/10.1093/cid/cis1020

15. Stover J, Rosen J, Kasedde S, et al. The impact and cost of the HIV/AIDS investment framework for adolescents. J Acquir Immune Defic Syndr 2014;66(Suppl 2):S170-S175. https://doi.org/10.1097/
and adolescents. J Acquir Im

16. Zimet GD, Liau A, Fortenberry VD. Health beliefs and intention to get immunized for HIV. J Adolesc Health 1997;20(5):354-359. https://doi.org/10.1016/S1054-139X(97)00031-1

17. Zealth 1997;20(5):354-359. https://doi.org/10.1016/S1054-139X(97)00031-1
Zimet GD, Mays RM, Fortenberry JD. Vaccines against sexually transmitted infections: Promise and problems of the magic bullets for prevention and control. Sex Transm Dis 2000;27(1):49-52.

8. Zimet GD, Mays RM, Winston Y, et al. Acceptability of human papillomavirus immunization. Womens Health Gend Based Med 2000;9(1):47-50. https://doi.org/10.1089/152460900318957

19. De Bruyn G, Skhosana N, Robertson G, McIntrye JA, Gray GE. Knowledge and attitudes towards HIV vaccines among Soweto adolescents. BMC Res Notes 2008;1:76. https://doi.org/10.1186/17560500-1-76

20. Jaspan HB, Berwick JR, Myer L, et al. Adolescent HIV prevalence, sexual risk, and willingness to participate in HIV vaccine trials. J Adolesc Health 2006;39(5):642-648. https://doi.org/10.1016/j. jadohealth.2006.05.016

21. Jaspan HB, Cunningham CK, Tucker TJ, et al. Inclusion of adolescents in prevention HIV vaccine trials: Public health policy and research design at a crossroads. J Acquir Immune Defic Syndr 2008;47(1):86-92. https://doi.org/10.1097/QAI.0b013e31815d2f27

22. Middelkoop K, Myer L, Mark D, et al. Adolescent and adult participation in an HIV vaccine trial

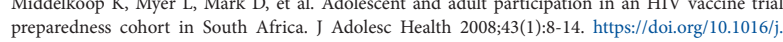
preparedness cohort in
jadohealth.2007.11.144

23. Otwombe KN, Sikkenma KJ, Dietricj J, de Bruyn G, van der Watt M, Gray GE. Willingness to participate in biomedical HIV prevention studies after the HVTN 503/Phambili trial: A survey conducted among adolescents in Soweto, South Africa. J Acquir Immune Defic Syndr 2011;58(2):211218. https://doi.org/10.1097/QAI.0b013e31822b7702 24. Smit J, Middelkoop K, Myer L, et al. Socio-behaviour challenges to phase III HIV vaccine trials in sub-
Saharan Africa. Afr Health Sci 2005;5(3):198-206. 
25. Smit J, Middelkoop K, Myer L, Seedat S, Bekker LG, Stein DJ. Willingness to participate in HIV vaccine research in a peri-urban South African community. Int J STD AIDS 2006;17(3):176-179. https://doi org/10.1258/095646206775809259

26. Ferrand RA, Corbett EL, Wood R, et al. AIDS among older children and adolescents in southern Africa: Projecting the time course and magnitude of the epidemic. AIDS 2009;23(15):2039-2046. https://doi.org/10.1097/QAD.0b013e32833016ce

27. Hargreaves JR, Morison LA, Kim JC, et al. The association between school attendance, HIV infection and sexual behaviour among young people in rural South Africa. J Epidemiol Community Health 2008;62(2):113-119. https://doi.org/10.1136/jech.2006.053827

28. Jewkes R, Nduna M, Levin J, et al. Impact of Stepping Stones on incidence of HIV and HSV-2 and sexual behaviour in rural South Africa: Cluster randomised controlled trial. BMJ 2008;337:a506. hexual behaviour in rural Sout

29. Sayles IN, Macphail CL, Newman PA, Cunningham WE. Future HIV vaccine acceptability among young adults in South Africa. Health Educ Behav 2010;37(2):193-210. https://doi.

30. Singh JA, Abdool Karim SS, Abdool Karim Q, et al. Enrolling adolescents in research on HIV and other sensitive issues: Lessons from South Africa. PLoS Med 2006;3(7):e180. https://doi.org/10.1371/ other sensitive issues: Lesso

31. Strode A, Slack C. Child privacy rights: A 'Cinderella' issue in HIV-prevention research. S Afr J HIV Med 2013;14(3):108-110. https:///oi.org/10.7196/SAJHIVMED.897

32. National Department of Health, South Africa. National HIV Counselling and Testing Policy Guidelines. Pretoria: NDoH, 2015. https://www.health-e.org.za/wp-content/uploads/2015/07/HCT-
Golicy Guidelines. Pretoria: NDoH, 2015. https://Www.h

33. Lau CY, Cardinali M, Sato PA, Fix A, Flores J. Broadening inclusion of vulnerable populations in HIV vaccine trials. Expert Rev Vaccines 2008;7(2):259-268. https://doi.org/10.1586/14760584.7.2.25

4. Slack CM, Strode AE. But is this really the 'parent' or 'guardian'? Practical strategies for consent to child

35. Strode A, Slack C. Sex, lies, and disclosures: Researchers and the reporting of under-age sex. S Afr HIV Med 2009;10(2):8-10. https://doi.org/10.4102/sajhivmed.v10i2.29

6. Strode A, Slack C, Essack Z. Child consent in South African law: Implications for researchers, service providers and policy-makers. S Afr Med J 2010;100(4):247-249.

77. Strode A, Slack C. Selected Ethical-legal Norms in Child and Adolescent HIV Prevention Research Consent, Confidentiality and Mandatory Reporting. Durban: European and Developing Countrie Trials Partnership (EDCTP), 2011
38. Bhamjee S, Essack Z, Strode E. Amendments to the Sexual Offences Act dealing with consensual underage sex: Implications for doctors and researchers. S Afr Med J 2016;106(3):356-259. https://doi. org/10.7196/SAMJ.2016.v106i3.9877

39. Shisana O, Rehle T, Simbayi LC, et al. South African National HIV Prevalence, Incidence and Behaviour Survey, 2012. Cape Town, HSRC Press, 2014.

40. Jaspan HB, Flisher AJ, Myer L, et al. Sexual health, HIV risk, and retention in an adolescent HIV prevention trial preparatory cohort. J Adolesc Health 2011;49(1):42-46. https://doi.org/10.1016/j. jadohealth.2010.10.009

41. Ellen JM, Wallace M, Sawe FK, Fisher K. Community engagement and investment in biomedical HIV prevention research for youth: Rationale, challenges, and approaches. J Acquir Immune Defic Syndr 2010;54(Suppl 1):S7-S11. https://doi.org/10.1097/QAL.0b013e3181e25779

42. Rubin RF, Kuttab HM, Rihani RS, Reutzel TJ. Patient adherence to three dose completion of the quadrivalent human papillomavirus (HPV) vaccine in a private practice. J Community Health 2012;37(6):1145-1150. https://doi.org/10.1007/s10900-012-9581-9

43. Widdice LE, Bernstein DI, Leonard AC, et al. Adherence to the HPV vaccine dosing intervals and factors associated with completion of 3 doses. Pediatrics 2011;127(1):77-84. https://doi.org/10.1542/ peds.2010-0812

44. Francis SA, Katz ML. The HPV vaccine: A comparison of focus groups conducted in South Africa and Ohio Appalachia. Matern Child Health 2013:17(7):1222-1229. https://doi.org/10.1007/s10995012-1116-6

45. Moodley N, Gray G. Global evidence reaffirms the case for routine HPV and potential HIV adolescent vaccination in South Africa. Future Virol 2014;9(2). https://doi.org/10.2217/fvl.13.131

46. Johnson LF, Bekker LG, Dorrington RE. HIV/AIDS vaccination in adolescents would be efficient and practical when vaccine supplies are limited. Vaccine 2007;25(43):7502-7509. https://doi.org/10.1016/j. vaccine, 2007.08.047

47. Bekker LG, Gray GE. Hope for the HIV control in southern Africa: The continued quest for a vaccine. PLoS Med 2017;14(2):e1002241. https://doi.org/10.1371/journal.pmed.1002241

Accepted 14 November 2017 